\title{
MAXIMAL ABELIAN SUBALGEBRAS IN HYPERFINITE FACTORS
}

\author{
BY OLE A. NIELSEN
}

Communicated by George Mackey, August 20, 1968

1. Introduction. In this note we outline a construction which, together with a new invariant, gives still more maximal abelian subalgebras of the hyperfinite factor of type $\mathrm{II}_{1}$. We also state some results concerning maximal abelian subalgebras of hyperfinite factors of type III. Complete proofs will appear elsewhere.

First we will establish some notation and terminology. Let $\mathfrak{T}$ and $\pi$ be maximal abelian subalgebras of a factor $a$. We call $\pi$ and $\pi$ equivalent (in $a$ ) if there is an automorphism of $a$ carrying $\mathscr{N}$ onto $\Re$. Let $N(\mathscr{T})=N^{1}(\mathscr{T})$ be the subalgebra of $a$ generated by all those unitary operators $U$ in $a$ with $U \Re U^{*}=\Re$, and let $N^{j}(\Re)$ $=N\left(N^{j-1}(\mathfrak{T})\right)$ for $j>1$. Following Dixmier [2] and Anastasio [1], we call $\Re$ regular if $N(\mathfrak{T})=Q$, semiregular if $N(\mathfrak{T C})$ is a factor distinct from $a$, and $n$-semiregular $(n \geqq 2)$ if no $N^{j}(\mathfrak{T}), 1 \leqq j<n$ is a factor but $N^{n}(\mathfrak{T l})$ is. We say that $\mathfrak{T}$ has proper [improper] length $n$ if $n$ is the smallest positive integer such that $N^{n}(\mathfrak{T})=N^{n+1}(\mathfrak{T})$ and if $N^{n}(\mathfrak{N})=a\left[N^{n}(\mathfrak{N}) \neq Q\right]$. Notice that the length of a maximal abelian subalgebra is invariant under equivalence. We are now able to state our main results.

ThEOREM 1. For each choice of $n=2,3$ and $k=0,1,2, \cdots$, the hyperfinite $\mathrm{II}_{1}$ factor contains an n-semiregular maximal abelian subalgebra of improper length $n+k$.

THEOREM 2. Let a be one of the hyperfinite type III factors of Powers (cf. [5]). Then a contains a regular and two inequivalent semiregular maximal abelian subalgebras. Also, for each choice of $n=2,3$ and $k$ $=0,1,2, \cdots$, a contains two n-semiregular maximal abelian subalgebras, one of proper length $n+k$ and one of improper length $n+k$.

REMARKs. (1) These results are a summary of the author's doctoral thesis written at the University of British Columbia under the supervision of Dr. D. Bures.

(2) Anastasio has shown that Theorem 1 holds with "improper" replaced by "proper" [1]. Because of a previous remark, our subalgebras are mutually inequivalent as well as inequivalent to those of Anastasio.

(3) $\Re$ has proper length $n$ if and only if $\mathscr{T}$ has length $n-1$ in the sense of Tauer [7]. 
(4) Pukánszky has given a general method for constructing maximal abelian subalgebras in a wide class of type III factors [6]; but, because of an error in the proof of Lemma 17, the types of these subalgebras is not known.

2. Construction of $\mathrm{II}_{1}$ factors. Let $G$ be a countably infinite group with identity $e$ and let $\Delta$ be the set of all functions with finite support from $G$ into $\{0,1\}$. Under point-wise addition modulo $2, \Delta$ becomes an abelian group. For $g \in G$ and $\alpha \in \Delta$, define elements $g \alpha$ and $\alpha g$ in $\Delta$ by

$$
\begin{aligned}
(g \alpha)(h) & =\alpha\left(g^{-1} h\right) & & (h \in G), \\
(\alpha g)(h) & =(\alpha(g))^{2}-2 \alpha(g)+1 & & h=g, \\
& =\alpha(h) & & \text { otherwise. }
\end{aligned}
$$

Let $H$ be a Hilbert space with orthonormal basis $\left(\phi_{\alpha}\right)_{\alpha \in \Delta}$. For each $g \in G$, define operators $F_{\theta}$ and $U_{\theta}$ on $H$ by

$$
\begin{aligned}
& F_{\theta} \phi_{\alpha}=\frac{1}{2} \phi_{\alpha}+\frac{1}{2} \phi_{\alpha \theta} \quad(\alpha \in \Delta), \\
& U_{0} \phi_{\alpha}=\phi_{0 \alpha} \quad(\alpha \in \Delta) \text {. }
\end{aligned}
$$

Notice that $\left\{F_{0}: g \in G\right\}$ is a commuting family of projections, that $g \rightarrow U_{g}$ is a unitary representation of $G$ on $H$, and that $U_{g} F_{h} U_{g}^{*}=F_{o h}$ for all $g, h \in G$. It is easy to verify that $\Re$, the von Neumann algebra on $H$ generated by $\left\{F_{g}: g \in G\right\}$, is maximal abelian in $\mathcal{L}(H)$ (use [3, p. 109, Exercise 5] and $\left.\phi_{0}\right)$. Let $B(G)$ be the von Neumann algebra on $H \otimes K$ generated by

$$
\left\{(M \otimes I)\left(U_{\theta} \otimes V_{\theta}\right): M \in \mathfrak{N}, g \in G\right\},
$$

where $K$ is the Hilbert space of all complex-valued square-summable functions on $G$ and $V_{g}$ is the unitary operator on $K$ satisfying $\left(V_{\theta} x\right)(h)$ $=x\left(g^{-1} h\right)$ for all $h \in G, x \in K$. Using some standard results from [3, pp. 127-137], one proves

LEMma 3. $\mathrm{B}(G)$ is a factor of type $\mathrm{II}_{1}$ which is hyperfinite whenever $G$ is the increasing union of a sequence of finite subgroups.

3. Subalgebras of $\mathrm{II}_{1}$ factors. For a subgroup $G_{0}$ of $G$, let $N\left(G_{0}\right)$ be the normalizer of $G_{0}$ in $G$ and let

$$
\operatorname{Tr}\left(G_{0}\right)=R\left(U_{0} \otimes V_{0}: g \in G_{0}\right) .
$$

Notice that $\Re\left(G_{0}\right)$ is always a proper subalgebra of $B(G)$. In the following three lemmas, $G_{\mathrm{c}}$ denotes a subgroup of $G$. 
Lemma 4 (CF. [6, Lemma 14]). $\Re\left(G_{0}\right)$ is maximal abelian in $B(G)$ if $\left\{g h g^{-1}: g \in G_{0}\right\}$ is infinite whenever $h \in G-G_{0}$.

PRoor. A straightforward calculation.

LEMMA 5. $\mathfrak{T}\left(G_{0}\right)$ is a factor if and only if all nontrivial conjugate classes of $G_{0}$ are infinite.

Proof. Observe that $\Re\left(G_{0}\right)$ is *-isomorphic to the group operator algebra over $G_{0}$, and apply [4, Lemma 5.3.4].

Lemma 6 (CF. [6, Lemma 17]). Suppose that $G_{0}$ satisfies: given a finite subset $F \subset G$ and a $g \in G$, there are infinitely many elements $g_{0} \in G_{0}$ such that

(i) $h, k \in F$ and $h g_{0} k^{-1}=g_{0}$ imply $h=k$,

(ii) if $g \notin N\left(G_{0}\right)$, then also $g_{0} g^{-1} \notin G_{0}$. Then $N\left(\mathfrak{T}\left(G_{0}\right)\right)=\Re\left(N\left(G_{0}\right)\right)$.

4. Proofs of the theorems. Applying the four preceding lemmas to the groups and subgroups used to prove Theorems I and II of [1], our Theorem 1 follows. The proof of Theorem 2, which is very involved technically, is based on the proof of [6, Lemma 17].

\section{REFERENCES}

1. S. Anastasio, Maximal abelian subalgebras in hyperfinite factors, Amer. J. Math. 87 (1965), 955-971.

2. J. Dixmier, Sous-anneaux abeliens maximaux dans les facteurs de type fini, Ann. of Math. (2) 59 (1954), 279-286.

3. - Les algebres d'operateurs dans l'espace Hilbertion, Gauthier-Villars, Paris, 1957.

4. F. J. Murray and J. von Neumann, On rings of operators. IV, Ann. of Math. (2) 44 (1943), 716-808.

5. R. T. Powers, Representations of uniformly hyperfinite algebras and their associated von Neumann rings, Bull. Amer. Math. Soc. 73 (1967), 572-575.

6. L. Pukánszky, Some examples of factors, Publ. Math. Debrecen 4 (1956), 135156.

7. Sister R. J. Tauer, Maximal abelian subalgebras in finite factors of type II, Trans. Amer. Math. Soc. 114 (1965), 281-308.

University of British Columbia, Vancouver 8, British Columbia, Canada 